\title{
Contactless Confined Readout of Quartz Crystal Resonator Sensors
}

\author{
Leonardo Steinfeld Volpe \\ Instituto de Ingeniería Eléctrica \\ Universidad de la República \\ Montevideo, Uruguay \\ leo@fing.edu.uy
}

Antonio Arnau Vives

Departamento de Ingeniería Electrónica

Universidad Politécnica de Valencia

Valencia, Spain

\author{
Marco Ferrari and Vittorio Ferrari \\ Dip. di Elettronica per l'Automazione and INFM \\ Università di Brescia \\ Brescia, Italy \\ marco.ferrari@unibs.it, vittorio.ferrari@unibs.it
}

\author{
Hubert Perrot \\ Laboratoire de Physique des Liquides et Electrochimie \\ CNRS, Université P. et M. Curie \\ Paris, France
}

\begin{abstract}
A configuration and method for contactless readout of the resonance response of a thickness-shear mode quartz resonator sensor is presented. The configuration uses a crystal with a large common electrode on the front face, and one, or more, small electrodes on the back face, leading to localized sensing regions. Each back electrode is capacitively coupled to a separated tip electrode. The tip consists of a small disc and a guard ring, which confine the electric field to the electrode area and make the measurement unaffected by the stray parallel capacitances. Analysis shows that the proposed configuration can be modeled as an extension of the Butterworth-Van Dyke (BVD) circuit and that, by a proper choice of the reference frequency around resonance, it is possible to obtain frequency readings that do not depend on the tip-to-crystal stand-off distance. Experimental results show that a mass load added on the front electrode can be consistently detected and measured irrespective of the tip-tocrystal stand-off distance.
\end{abstract}

\section{INTRODUCTION}

Multi-channel quartz crystal microbalances (QCM) have promising applications as sensors since they allow several QCM elements on one single crystal. A common problem with this approach is the interference between the elements due to cross-coupling of the acoustic field. Theoretical analyses based on Mindlin's theory [1] were proposed to study the influence of the electrode size and separation on frequency interference between two QCM on a monolithic quartz crystal [2]. The benefits of using mesa-shaped structures were analyzed [3]. The models of a QCM pair were constructed using finite element methods, and the effects of the design parameters, i.e. interval spacing between channels, electrode thickness, and the effect of the layout of QCM pair were investigated [4]. In general, there are evidences that the interference between adjacent QCMs of a single-crystal array can be minimized via the confinement of the energy trapping by properly choosing the different parameters or structures.

A further problem, however, remains regarding the difficulties in routing the electrical connections of the different elements of the array out of the crystal. This fact, apart from the interference issue, can pose practical limitations to the number of QCM elements that can coexist on a single crystal.

On the other hand, efforts have been made in doing twodimensional continuous mapping of a quartz surface with one common electrode and a scanning separated tip, attempting to resonate the quartz crystal only at a specific small region $[5,6]$. Although an acoustic load on the crystal could be detected via the scanning tip, a poor degree of spatial selectivity over the crystal surface resulted. Therefore, unsatisfactory results have been achieved in providing a quantitative and localized contactless scanning of the properties of a quartz crystal.

The present work proposes an improved contactless approach that obtains localized sensing regions via confined energy trapping under small electrodes placed on one of the crystal faces. The electrodes are capacitively coupled to a separated readout tip equipped with a guard electrode. The configuration avoids routing problems to the electrodes, at the same time minimizing the influence of stray contributions external to the crystal.

The proposed method may be instrumental towards the perspective development of multi-channel QCMs with contactless scanning. 


\section{THE SENSOR CONFIGURATION}

The configuration uses a quartz crystal with a common electrode on the front face and two or more electrodes on the back face. Each back electrode is capacitively coupled to a separated tip electrode. The resonance response is readout between the common front electrode and the tip that can be sequentially scanned across different back electrodes. The acoustic loads to be detected are placed on the front electrode in correspondence to each of the back electrodes. Due to the difference in area between the front and back electrodes, the geometry of the latter ones determine the energy trapping process, and hence the energy distribution profiles in the crystal [7]. A proper choice of the geometry and size of the back electrodes therefore leads to localized sensing regions.

Fig. 1 illustrates a configuration with two back electrodes. The figure shows the quartz crystal and the details of the tip. It can be observed that the tip consists of a small disc and an actively-driven guard ring. This provides the fundamental effect of confining the electric field to the electrode area, and it advantageously makes the measurement unaffected by the stray parallel capacitances.

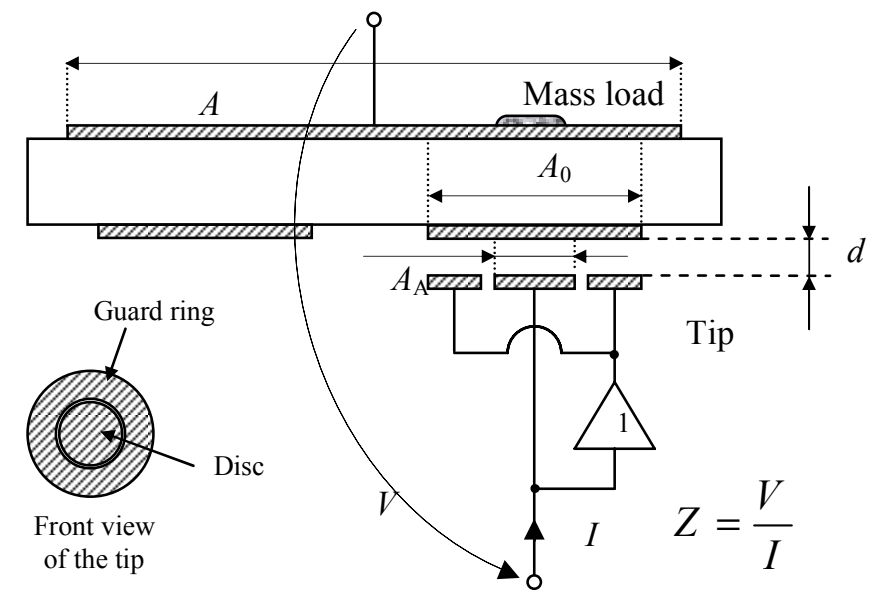

Figure 1. Schematic representation of the quartz crystal and the tip placed in front of one back electrode. The relevant parameters are: $d$ stand-off distance, $A_{\mathrm{A}}$ area of the disc, $A_{0}$ equivalent area of the resonator.

\section{THE MODEL}

The sensing properties of a QCM are a consequence of mechanical resonance in the crystal. The sensitive area is therefore dominated by mass distribution, typically defined by the size and thickness of the electrodes, not electric field distribution [8]. In our configuration of Fig. 1, despite the presence of the large front electrode, the mode trapping is due to the mass of the smaller back electrodes alone. Energy trapping is expected to confine the area of oscillation to the regions around the individual back electrodes, therefore leading to localized sensing regions as if they were separate resonators. In the simplest case where the resonators are decoupled, each of them can be modeled independently around the respective resonance as a Butterworth-Van Dyke (BVD) circuit [9]. As a consequence, the combination of each resonator with the tip-plus-guard assembly can be modeled as an extension of the Butterworth-Van Dyke (BVD) circuit, similar to that already proposed for a piezoelectric crystal with a separated electrode (PCSE) in electrochemical analysis [10]. Fig. 2 shows the extended BVD model where the additional series capacitor $C_{\mathrm{A}}$ accounts for the capacitance between the back electrode and the tip disc.

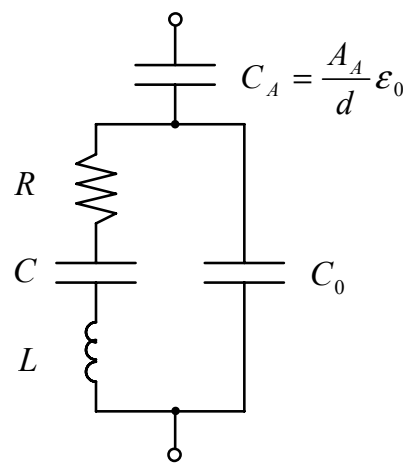

Figure 2. Extension of the BVD model where the capacitance $C_{\mathrm{A}}$ is added in series to simulate the effect of the capacitive coupling betwen the tip disc and the back electrode.

The motional parameters $R, L, C$ and the parallel capacitance $C_{0}$ only depend on the properties of the specific crystal region that defines the resonator. In particular, they depend on the area $A_{0}$ of the back electrode, and they do not depend on the tip stand-off distance $d$.

The capacitance $C_{\mathrm{A}}$ is the only parameter in the model that depends on the stand-off distance $d$. Since the guard ring steers the electric field parallel over the area $A_{\mathrm{A}}$, the expression of parallel plate capacitance can be assumed for $C_{\mathrm{A}}$, which is therefore proportional to $A_{\mathrm{A}} / d$.

An advantage provided by the guard ring is that the resonator is screened against parasitic parallel capacitances, therefore the model of Fig. 2 is complete and accurate.

\section{THE CHARACTERISTIC FREQUENCIES}

An acoustic load placed on the front electrode in correspondence to a given back electrode is expected to change the motional parameters in the model of Fig. 2. In general, the motional parameters could be extracted by fitting the model to the measured admittance, or impedance, curve. As an other approach, the model can be analyzed in order to individuate one or more characteristic frequencies that can be related to the relevant motional parameters through relationships as simple as possible.

The series resonant frequency $f_{s}^{*}$ of the resonatorguarded tip composite, defined as the frequency of the maximum of the real part of the admittance $Y(f)$, is given by:

$$
f_{s}^{*}=f_{s} \sqrt{\frac{C_{A}+C+C_{0}}{C_{A}+C_{0}}}
$$


where $f_{s}=\frac{1}{2 \pi \sqrt{L C}}$ is the series resonant frequency of the resonator alone. As $f_{s}^{*}$ depends on $C_{A}=\frac{\varepsilon_{0} A_{A}}{d}$, it varies with the stand-off distance $d$, therefore $f_{s}^{*}$ is not an optimal choice as a reference frequency.

On the other hand, the frequency $f_{p}^{*}$ of the composite, defined as the frequency of the maximum of the real part of the impedance $Z(f)$, does not depend on the series capacitance $C_{A}$. It can be shown that for low losses, so that $R^{2} \ll 2 L\left(C+C_{0}\right) / C C_{0}$, it follows that:

$$
f_{p}^{*}=f_{p}
$$

where $f_{p}=\frac{1}{2 \pi} \sqrt{\frac{C+C_{0}}{L C C_{0}}}$ is the parallel resonant frequency of the resonator alone. It can be observed that $f_{p}{ }^{*}$ only depends on parameters of the resonator. As such, $f_{p}^{*}$ can be advantageously adopted as a reference frequency unaffected by the stand-off distance $d$.

\section{THE QUARTZ CRYSTAL AND THE GUARDED TIP}

The experiments were performed on a $6 \mathrm{MHz}$ AT-cut quartz of $15-\mathrm{mm}$ diameter, with a large common top electrode of $13 \mathrm{~mm}$, and one off-center smaller back electrode of $4 \mathrm{~mm}$ in diameter, as shown in Fig. 3. The tip was fabricated on a printed circuit board. The disc is $2 \mathrm{~mm}$ in diameter and the external diameter of the guard ring is $4 \mathrm{~mm}$. The gap between disc and guard ring is $0.15 \mathrm{~mm}$.
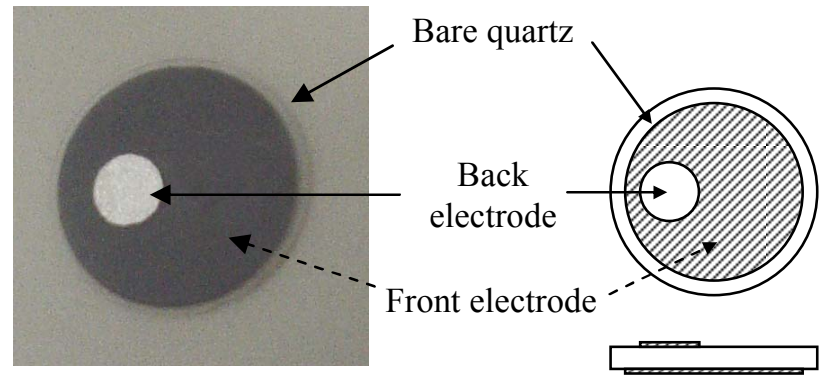

Figure 3. The quartz crystal resonator used in the experiments and the adopted electrode configuration.

\section{MEASUREMENT OF THE RESONANT FREQUENCIES}

The measurements of the resonator admittance $Y(f)$ and impedance $Z(f)$ with the driving of the guard ring according to the configuration of Fig. 1 were obtained by using an impedance analyzer (HP 4194A, Hewlett-Packard). The analyzer and quartz crystal were connected as shown in Fig. 4. Only the current flowing through the tip disc kept to virtual ground is measured, while the guard ring is tied to the actual ground terminal.

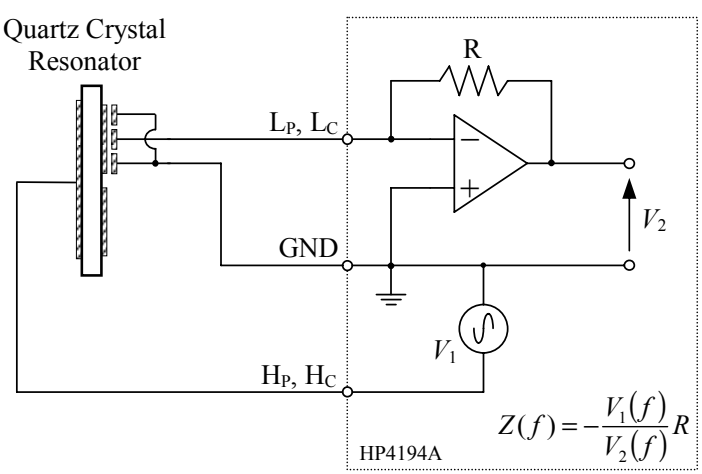

Figure 4. Configuration for the measurement of the resonator admittance and the driving of the guard ring using the virtual ground of the analyzer.

\section{EXPERIMENTAL RESULTS}

The guarded tip and the quartz crystal were mounted on a test fixture so that their relative position could be accurately set by a 2-D linear translation stage (Newport). In this way, measurements at varying the stand-off distance could be performed.

Fig. 5 shows the typical measured magnitude and phase of the impedance for the quartz crystal resonator shown in Fig. 3 at a tip stand-off distance of $10 \mu \mathrm{m}$.

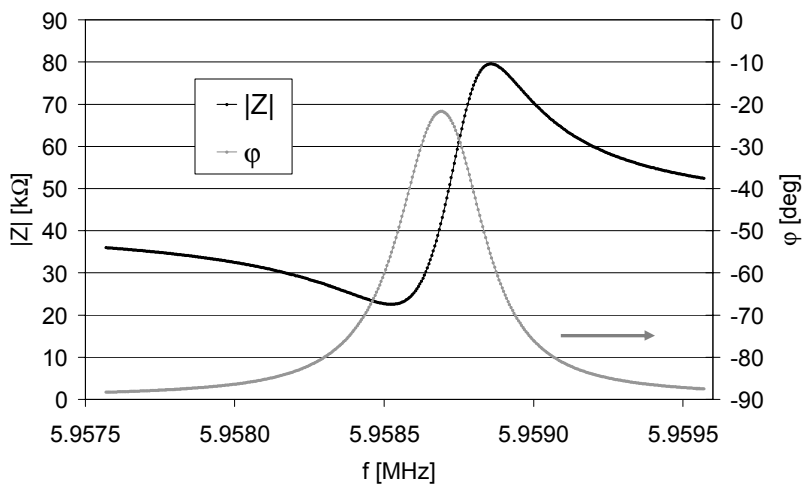

Figure 5. Typical measured impedance for the quartz crystal resonator shown in Fig. 3 at a tip stand-off distance. Of $10 \mu \mathrm{m}$.

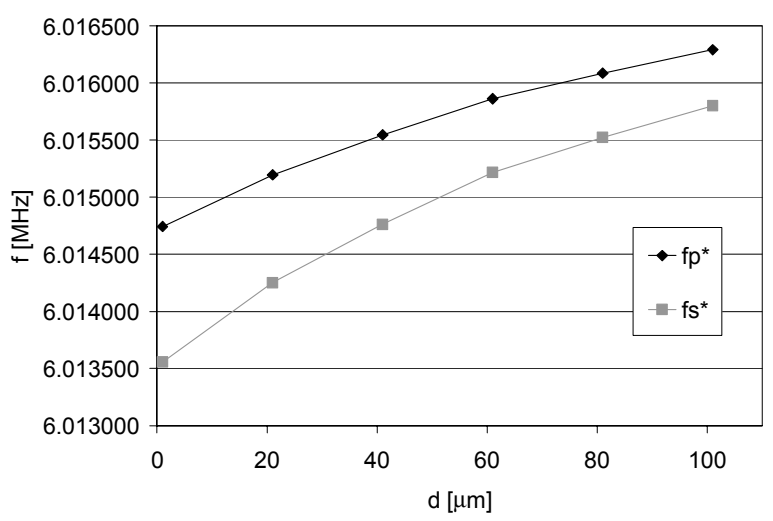

Figure 6. Measured frequencies $f_{p}^{*}$ and $f_{s}^{*}$ versus the stand-off distance of a quartz crystal without back electrode. 
At first, measurements were taken on a 6-MHz crystal without any back electrode. In such a case, all of the parameters in the model of Fig. 2 are expected to depend on the stand-off distance $d$ because of the variation of the electric and acoustic field distributions in the crystal with $d$.

According to the expectations, the results of Fig 6 show that both $f_{s}^{*}$ and $f_{p}^{*}$, defined as in paragraph IV, vary as the stand-off distance is increased.

Fig. 7 reports in the left-hand axis the measurement results obtained with the crystal with back electrode shown in Fig. 3. In this case it can be observed that $f_{s}^{*}$ varies with $d$, while $f_{p}^{*}$ remains constant, as expected from (1) and (2). A mass load represented by a minute paint dot, similar to what described in [11], was then added on the crystal front electrode in correspondence to the back electrode, and the frequency measurements versus $d$ were repeated. Fig. 7 in the right-hand axis shows that the measured frequency shifts $\Delta f_{s}^{*}$ and $\Delta f_{p}^{*}$ with respect to the unloaded condition were equal. As a consequence, there is evidence that $f_{p}^{*}$ can be used to detect mass irrespective of the distance $d$.

For the same mass load added off center, i.e. outside the region corresponding to the back electrode, no appreciable changes in frequency were measured. This demonstrates energy trapping, with a significant spatial confinement of the sensitivity to acoustic loading.

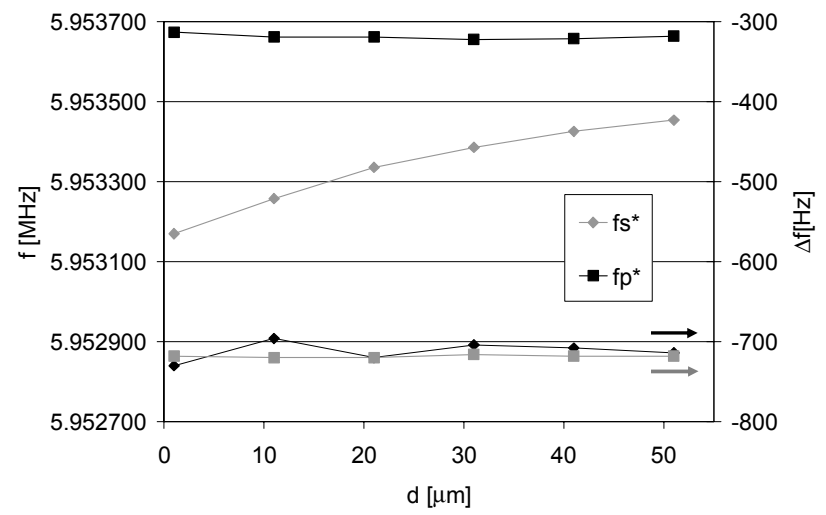

Figure 7. Measured frequencies $f_{p}{ }^{*}$ and $f_{s}{ }^{*}$ versus the stand-off distance (left-hand axis). Corresponding frequency shifts with respect to the unloaded values due to an added mass load (right-hand axis).

\section{CONCLUSION}

A configuration was proposed to excite and detect localized vibrations in a quartz crystal with a large front electrode by means of a contactless tip with active guarding that capacitively couples to a smaller back electrode. The configuration provides confinement of the electric and acoustic fields in the area of the crystal defined by the back electrode.

The configuration was modeled as an extended BVD circuit predicting that a specific reference frequency around resonance can be measured, i.e. $f_{p}{ }^{*}$, that is only related to the resonator motional parameters, while it does not depend on the tip-to-crystal stand-off distance.

Experimental results obtained with a 6-MHz crystal were in agreement with the predictions of the model. The measurement results showed that a mass load added on the front electrode, in correspondence to the back electrode, could be consistently detected, while for the same mass load added off center no appreciable changes in frequency were measured.

The proposed approach and configuration may be instrumental in obtaining independent QCM resonators over a monolithic quartz crystal, with the contactless readout that avoids routing problems in connecting the different electrodes.

\section{ACKNOWLEDGMENT}

L. S. V. thanks all the staff in the Electronics and Sensor Laboratory at the Università di Brescia for moral and technical support.

\section{REFERENCES}

[1] R. D Mindlin, P.C.Y. Lee, "Thickness-shear and flexural vibrations of partially plated crystal plates", Int. J. Solids Struct. 2 (1966) 125139.

[2] F. Shen, K. Hong Lee, S. J. O'Shea, P. Lu, and T. Yong Ng, "Frequency Interference Between Two Quartz Crystal Microbalances", IEEE Sensors Journal, Vol. 3, No. 3, June 2003, 274-281.

[3] F. Shen, P. Lu, S. J. O'Shea, and K. H. Lee, "Frequency coupling and energy trapping in mesa-shaped multi-channel quartz crystal microbalances", Sensors and Actuators A, 11 (2004), 180-187.

[4] F. Lu, H. P. Lee, P. Lu, S. P. Lim, "Finite element analysis of interference for the laterally coupled quartz crystal microbalances", Sensors and Actuators A, 119 (2005), 90-99.

[5] T. Tatsuma, K. Mori and N. Oyama, "Scanning Electrode Quartz Crystal Analysis. Back-Scanning Mode and Application to Electrochemical Measurements", Analitical Science, Vol.15 (1999), 749-753.

[6] M. Moulat, C. Gabrielli et H. Perrot, 202 ${ }^{\text {nd }}$ meeting ECS, 2002, Salt Lake City, USA.

[7] F. Josse, Y. Lee, S. J. Martin and R. W. Cernosek, "Analysis of the Radial Dependence of Mass Sensitiviy for Modified-Electrode Quartz Crystal Resonators", Anal. Chem. 70 (1998) 237-247

[8] P. J. Cumpson, "The quartz crystal microbalance: A new design eleimintates sensitivity outside the electrodes, often wrongly attributed to the electirc firing field"; Journal of Vacuum Science \& Techonogy A: Vaccum, Surfaces, and Films. July 1997 Volume 15 Issue 4 (1997), 2407-2412.

[9] "Piezoelectric Transducers and Applications", A. Arnau Vives Ed., 2004, Springer-Verlag, Berlin Heidelberg.

[10] S. Dazhong, N. Lihua and Y. Shouzhuo, "A new type of piezoelectric detector in liquid. Part 2: Computation of the equivalent circuit parameters of a piezoelectric crystal with a separated electrode and of series piezoelectric sensors in a non-electrolyte solution", J. Electroanal. Chem., 360 (1993) 71-87.

[11] P J Cumpson and M P Seah; "The quartz crystal microbalance: radialpolar dependence of mass sensitivity both on and off the electrodes"; Meas. Sci. Technol. 1 (1990) 544-555. 